\title{
Metabolome and Microbiome Signatures in the Roots of Citrus Affected by Huanglongbing
}

\author{
Emily M. T. Padhi, ${ }^{1}$ Nilesh Maharaj, ${ }^{2}$ Shin-Yi Lin, ${ }^{1}$ Darya O. Mishchuk, ${ }^{1}$ Elizabeth Chin, ${ }^{1}$ Kris Godfrey, ${ }^{3}$ Elizabeth Foster, ${ }^{3}$ \\ Marylou Polek, ${ }^{4}$ Johan H. J. Leveau, ${ }^{2}$ and Carolyn M. Slupsky ${ }^{1,5, \dagger}$ \\ ${ }^{1}$ Department of Food Science and Technology, University of California at Davis, Davis, CA 95616 \\ ${ }^{2}$ Department of Plant Pathology, University of California at Davis, Davis, CA 95616 \\ ${ }^{3}$ Contained Research Facility, University of California at Davis, Davis, CA 95616 \\ ${ }^{4}$ U.S. Department of Agriculture-Agricultural Research Service National Germplasm Repository, Riverside, CA 92507 \\ ${ }^{5}$ Department of Nutrition, University of California at Davis, Davis, CA 95616 \\ Accepted for publication 4 August 2019.
}

\begin{abstract}
Huanglongbing (HLB) is a severe, incurable citrus disease caused by the bacterium 'Candidatus Liberibacter asiaticus' (CLas). Although citrus leaves serve as the site of initial infection, CLas is known to migrate to and colonize the root system; however, little is known about the impact of CLas infection on root metabolism and resident microbial communities. Scions of 'Lisbon' lemon and 'Washington Navel' orange grafted onto 'Carrizo' rootstock were grafted with either CLas-infected citrus budwood or uninfected budwood. Roots were obtained from trees 46 weeks after grafting and analyzed via ${ }^{1} \mathrm{H}$ nuclear magnetic resonance spectroscopy to identify water-soluble root metabolites and high-throughput sequencing of 16S rRNA and ITS gene amplicons to determine the relative abundance of bacterial and fungal taxa in the root rhizosphere and
\end{abstract}

ABSTRACT endosphere. In both citrus varieties, 27 metabolites were identified, of which several were significantly different between CLas(+) and control plants. CLas infection also appeared to alter the microbial community structure near and inside the roots of citrus plants. Nonmetric multidimensional scaling (NMDS) and a principal coordinate analysis (PCoA) revealed distinct metabolite and microbial profiles, demonstrating that CLas impacts the root metabolome and microbiome in a manner that is variety-specific.

Keywords: bioinformatics, 'Candidatus Liberibacter asiaticus', citrus limon, citrus sinensis, huanglongbing, metabolomics, microbiome, plant-microbe interactions, plant pathogen interactions, roots
Huanglongbing (HLB), or citrus greening disease, is an infectious disease that is associated with the bacterium ' $\mathrm{Candi}$ datus Liberibacter asiaticus' (CLas) and is a serious threat to the sustainability of the citrus industry (McCollum and Baldwin 2016). CLas is transported systemically throughout the nutrientrich phloem tissue, where it colonizes sieve tubes and taps into resources meant for the plant. This eventually leads to phloem collapse, which severely disrupts nutrient transport (Kim et al. 2009). A key emerging component in HLB management is preserving root health as the pathogen is detected in root tissue as early as 2 months after infection, causing substantial degradation of the root system well before foliar symptoms appear (Johnson et al. 2014). Current management practices limit root loss by altering watering and fertilization methods; however, correctly

${ }^{\dagger}$ Corresponding author: C. M. Slupsky; cslupsky@ucdavis.edu

Current address of Shin-Yi Lin: Department of Food Science and Biotechnology, NCHU-UCD Plant and Food Biotechnology Center, and Agricultural Biotechnology Center, National Chung Hsing University (NCHU), Taichung 40227, Taiwan, ROC.

Funding: This study was supported by the California Citrus Research Board grant no. 5300-150. The salary for Shin-Yi Lin was provided by the Ministry of Science and Technology, Taiwan, Republic of China (NSC-102-2911-I-005-301, NSC-1032911-I-005-301) and the Ministry of Education, Taiwan, ROC, under the ATU plan. C.S. also acknowledges support through the U.S. Department of Agriculture National Institute of Food and Agriculture Hatch Project 1005945. Support for the Bruker Advance $600 \mathrm{MHz}$ NMR came from NIH grant RR011973.

*The $\boldsymbol{e}$-Xtra logo stands for "electronic extra" and indicates that two supplementary figures and six supplementary tables are published online.

The author(s) declare no conflict of interest.

Modified: 19 Feb 2020.

(C) 2019 The American Phytopathological Society diagnosing and removing infected trees is the most effective way to limit disease spread. The root system releases primary and secondary metabolites (termed 'root exudates') into the rhizosphere to serve as carbon/nitrogen substrates, signaling molecules, and chemoattractants for surrounding microbes (Hu et al. 2018), and plants shape the root microbiome by adjusting the root exudate composition, attracting beneficial microbes while deterring harmful ones (Lareen et al. 2016).

Metabolomics is an emerging technology that is able to examine changes in metabolism as an organism responds to an external stimulus, such as pathogen attack. Metabolomics has been used to quantify juice and fruit metabolites from HLB-affects citrus (Chin et al. 2014; Slisz et al. 2012), to examine the effects of Asian citrus psyllid (ACP) feeding on the citrus leaf metabolome (Chin et al. 2017), and to identify a metabolite fingerprint in CLas-infected citrus leaves (Cevallos-Cevallos et al. 2009) and root tissues (dos Santos Freitas et al. 2015). Similarly, microbiome studies of citrus have identified HLB-induced changes in bacterial community composition (Wang et al. 2017), including a shift toward bulk soil community composition with reductions in the populations of Burkholderia, Pseudomonas, Rhodanobacter, Sphingobium, and Sphingomonas (Trivedi et al. 2012). Others have reported similar results, with the depletion of Burkholderia and Bradyrhizobium observed in HLB-impacted sweet orange trees compared with healthy ones (Zhang et al. 2017). Conversely, Comamonadaceae, Hyphomicrobiaceae, and Rhizobiales appear to be enriched on the roots of infected trees (Blaustein et al. 2017). However, little is known regarding the metabolic response of citrus roots to CLas and the impact on surrounding microbial communities, and a combined analysis of the root metabolome and microbiome in response to a challenge by CLas has not been performed. Furthermore, no other research has compared how this response varies by cultivar. Therefore, the aim of this work was to characterize the response 
of two distinct varieties of citrus, 'Lisbon' lemon (Citrus limon L. Burm, f.) and 'Washington Navel' orange (Citrus sinensis (L.) Osbeck), grafted onto 'Carrizo' rootstock in terms of primary metabolites, bacteria, and fungi in the root system to ascertain the impact of host genotype and HLB infection on root-associated metabolism and microbial communities. We hypothesized that a distinct metabolite and microbial fingerprint would emerge for each variety based on health status, with different varietal responses to HLB.

\section{MATERIALS AND METHODS}

Plant materials. Roots were sampled from citrus trees $(n=34)$ that were part of a longitudinal experiment measuring metabolomics, microbiome, transcriptomics, and proteomics changes in the leaves of citrus trees affected by CLas (E. L. Chin, J. Ramsey, S. Saha, D. Mishchuk, J. Chaves, K. Howe, X. F. Zhong, M. FloresGonzalez, E. Mitrovic, M. L. Polek, K. Godfrey, L. A. Mueller, J. Bruce, M. Heck, and C. Slupsky, unpublished data). Trees were grown and maintained in an insect-free greenhouse in 2-gallon pots containing a 2:1 mixture of UC mix no. 2 and peat moss at the University of California (UC) Davis Contained Research Facility (Davis, CA, USA). The greenhouse was maintained at $27^{\circ} \mathrm{C}$ $\left( \pm 1.5^{\circ} \mathrm{C}\right)$ with supplemental lighting (high-pressure sodium lights) and a photoperiod of $16 \mathrm{~h}$ of light and $8 \mathrm{~h}$ of darkness. Humidity ranged from 30 to $50 \%$. Plants were watered as needed and fertilized each time with complete fertilizer (5-12-26) containing magnesium $(31 \mathrm{ppm})$, sulfate $\left(\mathrm{SO}_{4} 125 \mathrm{ppm}\right)$, iron $(3 \mathrm{ppm})$, manganese $(0.50 \mathrm{ppm})$, zinc (0.15 ppm), copper (0.15 ppm), boron $(0.50 \mathrm{ppm})$, molybdenum $(0.10 \mathrm{ppm})$, and calcium $(116 \mathrm{ppm})$. All experimental trees were scions of 'Lisbon' lemon (Citrus limon L. Burm, f.) or 'Washington Navel' sweet orange (Citrus sinensis (L.) Osbeck) grafted onto 'Carrizo' rootstock $(C$. sinensis $(\mathrm{L}$. $) \times P$. trifoliata $)$ that were grown from certified seed. Scions were grafted when rootstock were 6 months old, and they were allowed to grow for an additional 6 months. Seventeen trees (lemon, $n=11$; navel, $n=6$ ) were randomly assigned to be graftinoculated onto the varietal scion with three pieces of lemon or navel budwood of CLas (+) lemon or navel trees that were originally sourced from Hacienda Heights, CA. Seventeen trees (lemon, $n=11$; navel, $n=6$; control group) were grafted with uninfected budwood (lemon or navel). All trees were grown in the same soil and maintained under the same conditions.

Prior to grafting, the abundance of CLas in infected source trees was confirmed by a quantitative PCR (qPCR), as previously described (Li et al. 2006), and expressed as a cycles to threshold (Ct) value for each sample. Similarly, the presence of the pathogen in experimental trees was quantified by performing qPCR for leaf samples that were randomly sampled throughout the canopy starting 10 weeks after grafting. For this, six to eight leaves per tree were collected, of which $200 \mathrm{mg}$ (fresh weight) of midrib and petiole tissue was excised and used for qPCR as per the United States Department of Agriculture (USDA) Animal and Plant Health Inspection Service (APHIS) Plant Protection and Quarantine (PPQ) program protocol using the Qiagen MagAttract plant DNA extraction kit (Valencia, CA). Leaf sampling for metabolomics in the parent study commenced 10 weeks after exposure and was performed approximately every 6 weeks thereafter for a total of seven times between February 2014 and January 2015 (E. L. Chin, J. Ramsey, S. Saha, D. Mishchuk, J. Chaves, K. Howe, X. F. Zhong, M. Flores-Gonzalez, E. Mitrovic, M. L. Polek, K. Godfrey, L. A. Mueller, J. Bruce, M. Heck, and C. Slupsky, unpublished data). Visual symptoms of HLB on the canopy of experimental plants were monitored throughout the study, and visual symptoms of the roots were recorded at the final time point (January 2015; 46 weeks after exposure). A tree with a $\mathrm{Ct}$ value $<37$ at least once during the study was considered to be infected with CLas (USDA-APHIS 2013). Root samples were collected by snipping feeder roots from each quadrant of the bulk root mass of each individual tree using stainless steel surgical scissors that were sterilized with $70 \%$ ethanol between sample collections. These subsamples were then pooled into a single sample.

Sample preparation and data collection. To perform a microbial analysis of the pooled root samples, roots were shaken to remove loosely adhering soil particles. Up to $3 \mathrm{~g}$ of root tissue was transferred to a $50 \mathrm{ml}$ conical tube and filled with wash buffer (20 mM Tris- $\mathrm{HCl}, 10 \mathrm{mM}$ EDTA, and $0.024 \%$ Triton) (Rastogi et al. 2010) to an adjusted volume of $45 \mathrm{ml}$. The tube was vortexed for $20 \mathrm{~s}$, sonicated in a Branson water bath for $7 \mathrm{~min}$, and vortexed again for $20 \mathrm{~s}$. The root material was removed, and the root wash was centrifuged for $7 \mathrm{~min}$ at 7,197 $\times g$. The supernatant was discarded and the pellet was used for DNA extraction. The root endosphere community was extracted by washing the roots twice more and grinding them in liquid nitrogen into a fine powder using a mortar and pestle. Rhizosphere soil DNA extractions were performed using the PowerSoil DNA Isolation Kit (cat. \#12888; Mo Bio Labs) using approximately $250 \mathrm{mg}$ of soil per extraction. Root endosphere DNA extractions were performed using the PowerPlant Pro DNA Isolation Kit (cat. \#13400-50; Mo Bio Labs) using approximately $60 \mathrm{mg}$ of tissue per extraction. Microbial DNA extracts from rhizosphere soils or root tissues were diluted 10 -fold and used as template during PCR to amplify the V5-V7 region of the bacterial $16 \mathrm{~S}$ ribosomal gene using primers $799 \mathrm{~F}$ and 1193R (Bodenhausen et al. 2013; Chelius and Triplett 2001) or the ITS1 (intergenic spacer region 1) region using primers BITS1 and B58S3 (Bokulich and Mills 2013). The 799F and BITS forward primers contained built-in barcodes (Aleklett et al. 2015; Hamady et al. 2008). PCRs were performed using GoTaq Green Master Mix (cat. \#M7122; Promega) in 15- $\mu$ l reactions containing $7.5 \mu \mathrm{l}$ of $2 \mathrm{X}$ GoTaq Green master mix, $3.9 \mu \mathrm{l}$ of water, $0.3 \mu \mathrm{l}$ of reverse primer $(10 \mu \mathrm{M}), 0.3 \mu \mathrm{l}$ of bovine serum albumin (BSA) $(20 \mathrm{mg} / \mu \mathrm{l}$; NEB cat. \#B9000S), $1.5 \mu \mathrm{l}$ of forward primer $(2 \mu \mathrm{M})$, and $1.5 \mu \mathrm{l}$ of template DNA. Cycling conditions were as follows: initial denaturation at $94^{\circ} \mathrm{C}$ (bacterial) or $95^{\circ} \mathrm{C}$ (fungal) for $2 \mathrm{~min}$, then 35 cycles of denaturation at $94^{\circ} \mathrm{C}$ (bacterial) or $95^{\circ} \mathrm{C}$ (fungal) for $30 \mathrm{~s}$, primer annealing at $55^{\circ} \mathrm{C}$ for $30 \mathrm{~s}$, product extension at $72^{\circ} \mathrm{C}$ for $1 \mathrm{~min}$, and final extension at $72^{\circ} \mathrm{C}$ for $10 \mathrm{~min}$ (bacterial) or 5 min (fungal). Amplicons were visualized on a $1 \%$ agarose gel, pooled, and, in the case of bacterial amplicons, run again on a $0.6 \%$ gel to achieve separation of the 430-bp nonmitochondrial band that was excised from gel and purified using the Zymoclean Gel DNA Recovery Kit (cat. \#D4007; Zymo Research). The mitochondrial product was $\approx 800 \mathrm{bp}$. The fungal amplicon pool was purified using the QIAquick PCR Purification Kit from Qiagen (cat. \#28104). Bacterial and fungal amplicon pools were submitted for library preparation and sequencing at the UC Davis DNA Technologies core, where sequencing was performed using the IIlumina MiSeq platform with the 300 base pair (bp) pairedend read platform.

For metabolomics, root samples were washed with deionized water to remove attached soil, blotted dry with paper towels, and stored in sealable plastic bags at $-80^{\circ} \mathrm{C}$. Approximately $1 \mathrm{~g}$ of frozen root sample was lyophilized in a Falcon $15 \mathrm{ml}$ conical centrifuge tube using a Labconco FreeZone Plus 4.5-liter freeze-dry system (Kansas City, MO, USA) for $48 \mathrm{~h}$. Tubes containing dry roots were subsequently placed in a resealable bag and stored at room temperature and protected from light until metabolite extraction. Dried root samples were cut into small pieces using stainless steel surgical scissors, and $\approx 75 \mathrm{mg}$ was transferred to $2-\mathrm{ml}$ screw-cap microcentrifuge tubes. One 3.5-mm-diameter glass bead (BioSpec Products Inc., Bartlesville, OK, USA) was placed in each tube and ground for 2 min using a BioSpec Mini-Beadbeater-16 cell disrupter (BioSpec Products Inc., Bartlesville, OK, USA), after which $10 \mathrm{mM}$ of potassium phosphate buffer $(\mathrm{pH} 6.8 \pm 0.1)$ was added to each tube at a ratio of 1:20 wt/vol. Samples were then incubated in an Eppendorf Thermomixer $\left(15 \mathrm{~min}, 90^{\circ} \mathrm{C}, 1000 \mathrm{rpm}\right)$ and centrifuged $\left(15 \mathrm{~min}, 4^{\circ} \mathrm{C}, 14,000 \mathrm{RCF}\right)$ using an Eppendorf Model 5415R microcentrifuge (Eppendorf North America, 
Hauppauge, NY, USA). An aliquot (1000 $\mu$ l) of the supernatant was transferred to a new tube, to which $100 \mathrm{mg}$ of BioRad Chelex 100 resin in sodium form (BioRad, Hercules, CA, USA) was added; the mixture was allowed to stand for $15 \mathrm{~min}\left(4^{\circ} \mathrm{C}\right)$. Samples were centrifuged $\left(15 \mathrm{~min}, 4^{\circ} \mathrm{C}, 14,000 \mathrm{RCF}\right)$, and $400 \mu \mathrm{l}$ of supernatant was transferred to an Amicon Ultra 0.5-ml centrifugal filter unit with an Ultracel-3 membrane (EMD Millipore, Billerica, MA, USA) that was prewashed $(4 \times)$ with purified water and centrifuged again $\left(30 \mathrm{~min}, 4^{\circ} \mathrm{C}, 14,000 \mathrm{RCF}\right)$. From this, $207 \mu \mathrm{l}$ of filtrate was combined with $23 \mu \mathrm{l}$ of internal standard containing $5 \mathrm{mM} \mathrm{3-}$ (trimethylsilyl)-1-propanesulfonic acid-d6 (DSS- $\mathrm{d}_{6}$ ), $\mathrm{NaN}_{3}$, and $\mathrm{D}_{2} \mathrm{O}$ (Chenomx, Edmonton, $\mathrm{AB}$, Canada). The final concentrations were $0.5 \mathrm{mM} \mathrm{DSS}-\mathrm{d}_{6}, 0.02 \% \mathrm{NaN}_{3}$, and $\approx 10 \% \mathrm{D}_{2} \mathrm{O}$. The $\mathrm{pH}$ of each sample was adjusted to $6.8 \pm 0.1$ using small amounts of $1 \mathrm{~N}$ $\mathrm{HCl}$ or $\mathrm{NaOH}$, and $180 \mu \mathrm{l}$ was transferred to $3-\mathrm{mm}$ nuclear magnetic resonance (NMR) tubes that were stored at $4^{\circ} \mathrm{C}$ until NMR data were acquired (within $24 \mathrm{~h}$ of sample preparation).

Metabolomics analysis. NMR spectra were acquired at $298 \mathrm{~K}$ using the Bruker 'noesypr1d' experiment on a Bruker Avance 600 $\mathrm{MHz}$ NMR spectrometer equipped with a SampleJet. The acquisition parameters were as follows: sweep width, $12 \mathrm{ppm}$; acquisition time, $2.5 \mathrm{~s}$; relaxation delay, $2.5 \mathrm{~s}$; and mixing time, $100 \mathrm{~ms}$. Water saturation was applied during the relaxation delay and mixing time. The resulting spectra were zero-filled to 128,000 data points, and an exponential apodization function corresponding to a line-broadening of $0.5 \mathrm{~Hz}$ was applied. Spectra were processed for metabolite identification and quantification using the Chenomx Inc. NMR Suite Processor version 8.2 (Edmonton, $\mathrm{AB}$, Canada).

Microbiome analysis. Illumina DNA sequences from bacteria and fungi were processed and analyzed using QIIME version 1.9.1 (Caporaso et al. 2010). For the bacterial community, paired reads were joined using join_paired_ends.py; barcodes were extracted using extract_barcodes.py; reads were quality-filtered and demultiplexed using split_libraries_fastq.py -q 19; OTUs (operational taxonomic units) picked at $97 \%$ similarity using pick_open_ reference_otus.py $-\mathrm{s} 0.1$; the OTU table was filtered to remove OTUs that constituted $<0.005 \%$ of all reads using filter_otus_from_ otu_table.py-min_count_fraction 0.00005; and the OTU table was filtered again to remove chloroplast OTUs using filter_taxa_from_ otu_table.py -n c__Chloroplast,f__mitochondria. For the fungal community, sequence processing was performed as previously described with the following exceptions: the paired ends were not joined and taxonomy was assigned using the RDP classifier with the UNITE database (ver. 6, 2014-09-10, developer files) (Kõljalg et al. 2013). Beta diversity comparisons were performed using jackknifed_ beta_diversity.py with the Bray-Curtis dissimilarity and the weighted and unweighted unifrac (bacteria only) metrics. Cluster significance was tested using the Adonis function, an implementation of Permutational MANOVA (Anderson 2001), in compare_ categories.py. Core OTUs were calculated using compute_core_ microbiome.py.

Statistics and data analysis. Root metabolite concentrations and microbial abundance data in roots were evaluated for normality using histograms and the Shapiro-Wilk test. Metabolite concentrations were corrected for dilution and converted to nmol/g of dry leaf weight. Differences in metabolite concentration and microbial abundance between healthy and CLas $(+)$ plants were compared using the Mann-Whitney $U$ test, and results were considered significant if $P<0.05$. The application of a false discovery rate (FDR) resulted in nonsignificant findings for all microbial data. Therefore, significant unadjusted $P$ values, in combination with large effect sizes, were used to interpret the results. Absolute median $\log _{2}$ fold-change values in metabolites between CLas(+) and control groups were calculated, with negative fold changes representing a reduction in metabolite concentrations with exposure to CLas. Effect sizes were estimated with Cliff's delta $(d)$ using the following calculation:
$\operatorname{ABS}(2 * U) / n_{a} n_{b}-1$

where $U$ represents the calculated Mann-Whitney $U$ statistic and $n_{\mathrm{a}} n_{\mathrm{b}}$ represents the product of the control and CLas+ samples sizes (Goedhart 2016). Effect sizes were interpreted as follows: $d<0.147$, negligible; $d<0.33$, small; $d<0.474$, medium; and $d>0.474$, large (Romano et al. 2006). Associations between metabolites or microbes and qPCR $\mathrm{Ct}$ values from leaves were examined using the Spearman rank correlation ('HMisc' package; R), and results were considered significant if the FDR-adjusted (BenjaminHochberg) results had $P<0.05$. Shifts in the root metabolome and microbiome were compared using a permutational multivariate analysis of variance (PERMANOVA) of cluster centers (termed centroids) on the Bray-Curtis distance matrix created via nonmetric multidimensional scaling (NMDS) and principal coordinate analysis (PCoA), respectively ('vegan' and 'phyloseq' packages; R). Graphs were produced and statistical procedures were performed using R version 3.5.1 (R Core Team 2012).

Accession numbers. Sequencing data will be deposited in the European Nucleotide Archive.

\section{RESULTS}

Metabolic and microbiome signatures of healthy control rootstocks grafted with lemon or navel scions. A comparison of roots from control (healthy) trees revealed significant differences in a small number of metabolites in lemon roots compared with navel (Supplementary Table S1); however, the NMDS analysis of the centroids of each group revealed that the metabolomes of lemon and navel controls were not significantly different (Supplementary Fig. S1). NMDS is an ordination method that configures points in two-dimensional or three-dimensional space, where each point represents individuals characterized by the data collected, and in which the distances between individual points are calculated dissimilarity values (Cox and Ferry 1993). PERMANOVA utilizes a pseudo $F$ statistic to test the null hypothesis that there is no difference in the position of the group centroids (the center of the clusters) given by the selected dissimilarity measure (Anderson 2001). Differences were not expected because samples were collected $\approx 18$ months after scions were fused onto rootstock, allowing sufficient time for vascular tissues to fuse (Melnyk 2017). This was in contrast to differences observed in the bacterial and fungal communities measured in and around the root system of healthy lemon and navel plants, which suggested unique microbial structures are formed in the root systems of citrus plants and are shaped by the varietal scion (Supplementary Table S2). These differences were reflected in varietal clustering apparent in PCoA plots (Supplementary Fig. S2). At the phylum level, roots of healthy lemons harbored more Proteobacteria and Acidobacteria than roots of healthy navels, less Actinobacteria, and more of the fungal phylum Basidiomycota. At the family level, lemons were dominated by Burkholderiaceae (represented largely by Burkholderia). In contrast, navels contained significantly more Microbacteriaceae, Patulibactereaceae, and Rhizobiaceae compared with lemons. Fungal signatures distinguishing lemon and navel roots were also observed; in order of greatest abundance, Herpotrichiellaceae, Nectriaceae, and Entolomataceae dominated in lemons, and Nectriaceae, Trichocomaceae, Pleosporaceae, Microascaceae, and Herpotrichiellaceae were dominant in navels.

Infection status and visual symptoms of CLas graftinoculated trees. Among 17 citrus trees graft-inoculated with budwood originating from CLas(+) trees, 6 of 11 lemon and 4 of 6 navel trees were confirmed by qPCR to contain a sufficient bacterial titer to warrant a positive CLas classification (i.e., $\mathrm{Ct}<37$ ). The remaining plants in the graft-inoculated group (5 lemon, 2 navel) did not reach this threshold; therefore, these plants were considered CLas(-) and were removed from the analysis (Supplementary Table $\mathrm{S} 3$ ). The $\mathrm{Ct}$ values of infected lemon plants at week 46 ranged from 
23.30 to 25.65 and from 19.38 to 22.63 among infected navel plants. All control trees tested negative for CLas, with the exception of two navel controls, which had $\mathrm{Ct}<37$ at one time point during the study period. After retesting, the same samples were determined to have a $\mathrm{Ct}$ of 40; however, we did not include these two trees in the final statistical analyses because of uncertainty. Because of a limitation during sample collection, root materials for microbial analyses were randomly collected for only 6 of 11 lemon control plants, resulting in a total of 20 trees for microbial community analysis: 10 CLas(+) trees (6 lemon, 4 navel) and 10 CLas(-) (control) trees (6 lemon, 4 navel). Roots obtained from CLas $(+)$ plants at 46 weeks after exposure were visually distinguishable from control plants, especially in terms of bulk root mass, which was substantially less in CLas(+) plants. Other visual symptoms of HLB included mottling, yellowing, and curling of the plant leaves (Fig. 1). Trees that were CLas(+) in leaf tissue also presented with detectable CLas in the roots.

Metabolite signature of CLas(+) citrus roots. Of approximately 320 compounds in the Chenomx metabolomics database, 27 water-soluble metabolites were identified in citrus roots, including sugars, amino acids, organic acids, and other compounds related to plant metabolism and defense. This method enabled us to examine changes in several primary metabolites that are central to metabolism, including amino acids, sugars, and organic acids. The NMDS analysis of citrus root metabolites revealed four clusters related to the variety and infection status (Fig. 2). CLas-induced changes in citrus roots included a significant reduction in the concentration of 15 metabolites in lemons and 3 metabolites in navels, which were accompanied by large effect sizes for each significant metabolite. Additionally, several metabolites in navel roots (sucrose, asparagine, malic acid, quinic acid, choline, trigonelline) did not reach statistical significance, but they did have a large effect size. Compared with standard null hypothesis significance testing, which indicated whether an experimental effect is caused by chance (Middlemis Maher et al. 2013), effect sizes allowed the magnitude of the effect to be examined, thereby providing an indicator of biological significance (Nakagawa and Cuthill 2007).

Overlap in the plant response to CLas was observed between varieties, including a reduction in all sugars measured, the amino acids proline and asparagine, the metabolites choline and uridine, and an increase in trigonelline. Interestingly, there were some differences in the varietal responses to disease. For example, the metabolites malic acid and quinic acid were significantly reduced in lemon roots but appeared to increase in navel roots (nonsignificant results, but large effect sizes) (Fig. 3). Correlation analyses were conducted to examine relationships between bacterial titers of CLas (lowest reported Ct value in leaf tissue; Supplementary Table S4) and root metabolites. Lower $\mathrm{Ct}$ values represent greater proliferation of the bacterium in planta. In lemon roots, higher concentrations of sugars (glucose, maltose, and sucrose) and some amino acids (alanine, asparagine, proline, threonine, and GABA) correlated with higher CLas Ct values. Additionally, as the Ct value decreased, there appeared to be a trend toward an increase in the trigonelline concentration (results not significant after BenjaminiHochberg FDR correction). These findings agreed with the difference testing conducted during this study that showed higher concentrations of these key metabolites in CLas(-) lemon plants relative to CLas(+) lemon plants.

Microbial signature of CLas(+) in citrus roots. Overall, 1,002 bacterial and 409 fungal OTUs were identified in root soil (rhizosphere) and root tissue (endosphere) samples combined. The core bacterial and fungal microbiomes of the rhizosphere and endosphere of Lisbon lemon and Washington Navel orange trees were summarized at the family and genus levels and were defined as at least one sequencing read in $\geq 75 \%$ of samples in a group (Blaustein et al. 2017) [i.e., present in the roots of 5 of 6 plants in the lemon control and CLas(+) groups and in 3 of 4 plants belonging to the navel control and CLas(+) groups]. Figure 4 shows the 10 most abundant dominant bacteria and fungi (described at the family and genus levels). The majority of dominant bacteria were also members of the core bacterial microbiome ( 9 of 10 families, 8 of 10 genera). Several differences in bacterial and fungal community compositions were observed in CLas(+) plants compared with controls; however, interestingly, there was no overlap in these difference between citrus varieties.

The navel response to CLas was characterized by large increases in the relative abundance of Burkholderiaceae and Burkholderia in both the endosphere and rhizosphere, a dramatic decrease in Enterobacteriaceae in the rhizosphere, an increase in representatives from the fungal genus Exophiala, and changes in the relative abundance of several other less dominant microbes (Fig. 5, Supplementary Table S5). Conversely, the lemon response to CLas was characterized by large changes in fewer microbes. A large increase in the bacterial family Xanthomonadaceae was observed in both the endosphere and rhizosphere of lemons, along with a

CLas(+)

Control
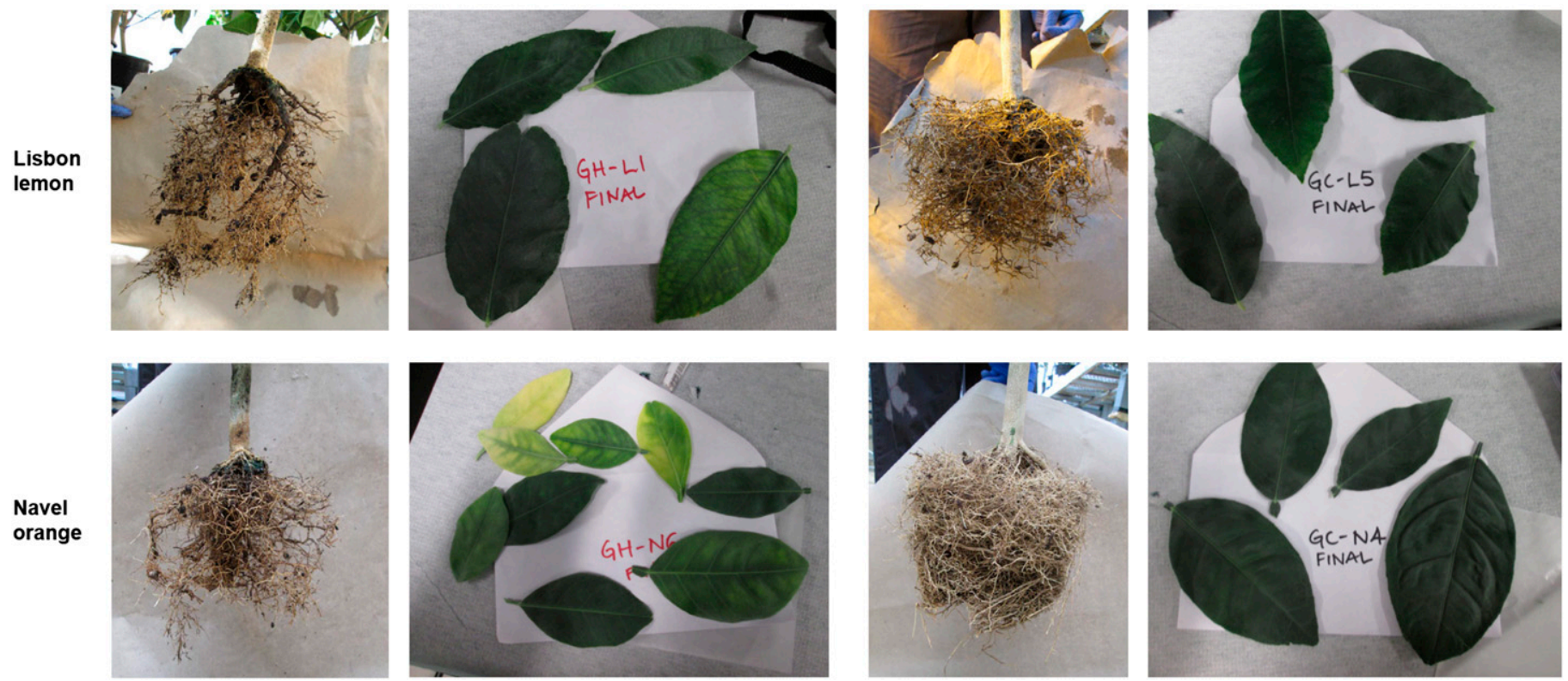

Fig. 1. Images of the bulk root mass and sample leaves from healthy and 'Candidatus Liberibacter asiaticus' lemon and navel plants. 
dramatic decrease in the fungal genus Exophiala and an increase in the bacteria Scytaldium and Penicillum. Similar to navel roots, Burkholderia species were dominant, but the abundance of this genus did not change with infection in lemons. The fungi Hypocrea increased significantly in both the endosphere and rhizosphere of lemons, and a large effect size was observed in the navel rhizosphere. The PCoA of microbial communities in the lemon and navel endosphere and rhizosphere showed a significant separation of samples based on the infection status (Fig. 6). Correlation analyses revealed several significant correlations between bacterial titers of the least reported CLas Ct value in leaf tissue and microbial communities (Supplementary Table S6), but these findings were mostly nonsignificant after FDR correction at all sites measured except the navel rhizosphere. Here, strongly significant negative correlations were observed between CLas Ct values of leaf tissue and the bacterial classes Betaproteobacteria and Solibacteres, the family Methylocystaceae, and the genus Burkholderia, and a strongly positive correlation was observed for the class Gammaproteobacteria. Additionally, fungi in Ascomycota were strongly and positively correlated with CLas $\mathrm{Ct}$ values, whereas a strong negative correlation was observed with Basidiomycota. No significant correlations were observed in lemon roots.

\section{DISCUSSION}

Summary of findings. The aim of this research was to characterize metabolic and microbial shifts in lemon and navel roots affected by HLB. Differences in key metabolites, bacteria, and fungi were evident between healthy and diseased plants. Interestingly, host responses to CLas were strongly influenced by variety, despite the use of identical 'Carrizo' rootstock. A recent study showed that alterations in root metabolite concentrations of citrus occur when scions are grafted onto different rootstock, but that the extent of these alterations depends on the variety of citrus examined (Albrecht et al. 2019). Here, we found that the scion variety did not significantly alter the root metabolome in healthy plants 18 months after grafting. The opposite was observed in microbial communities; significant differences were found between lemon and navel controls. This suggested that the scion variety strongly influences root microbial communities, but not root metabolites in citrus. If root exudates are primary drivers of the microbial community composition (Hu et al. 2018), then our findings suggest transient changes in metabolites are sufficient to alter community structure. Alternatively, these findings could suggest that the microbial structure is not impacted by water-soluble

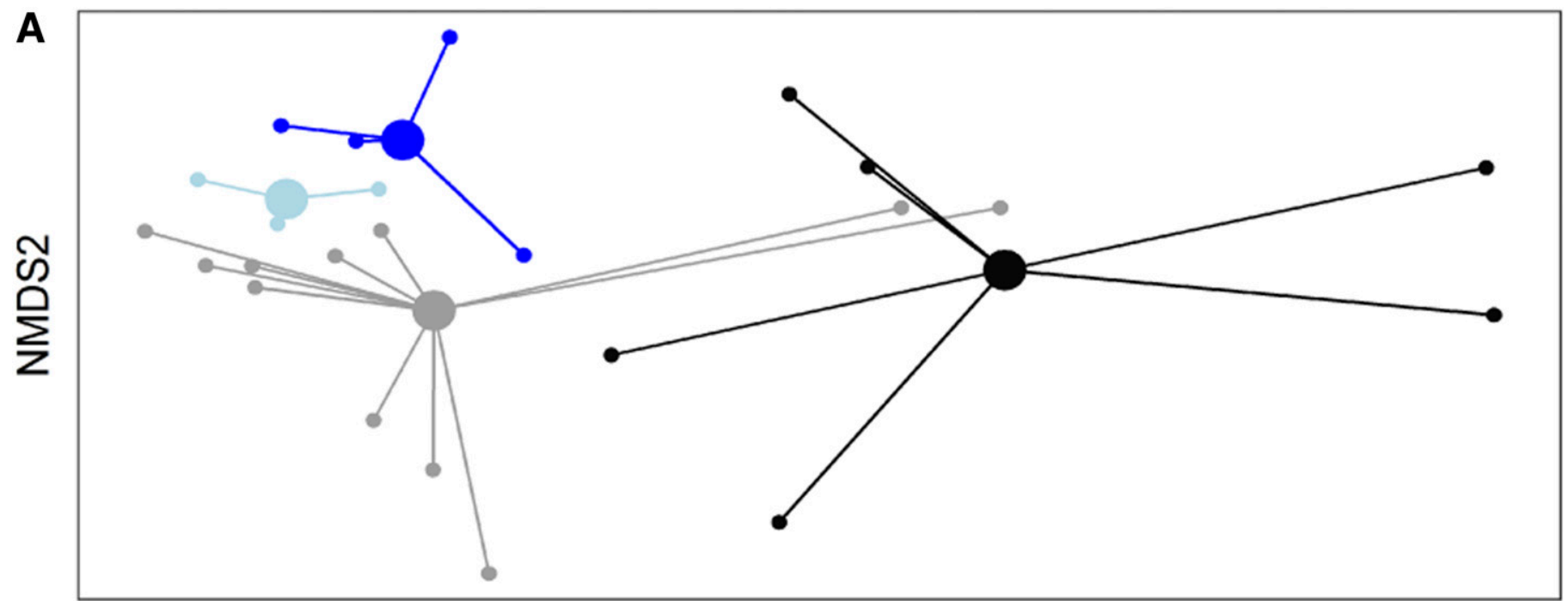

NMDS1

B

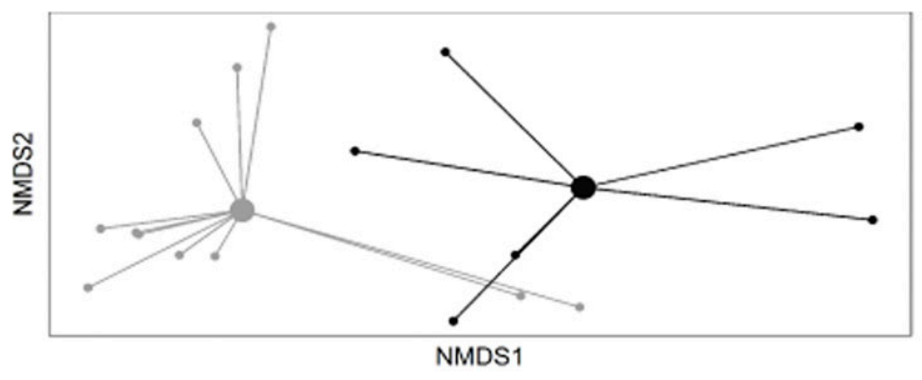

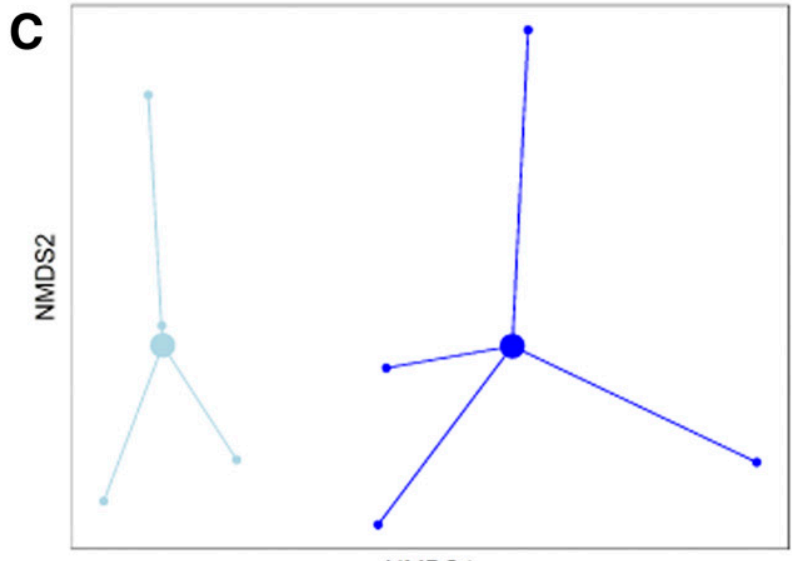

NMDS1

Fig. 2. Nonmetric multidimensional scaling (NMDS) ordinations based on Bray-Curtis dissimilarity distances of metabolite concentrations in citrus roots. Plots show variations in the root metabolome by variety and infection status ('Candidatus Liberibacter asiaticus' [CLas] versus healthy). Each small circle represents a single tree. The large circles represent the centroid (the weighted average location) of each sample type. Points plotted closer together indicate similar metabolic compositions. Low stress values $(<0.20)$ indicate the model is representative of sample distances in two dimensions. A, Greenhouse-grown citrus trees (PERMANOVA, $R^{2}=0.42, P=0.0010$, stress $=0.100$ ). B, Lisbon lemon (PERMANOVA, $R^{2}=0.33, P=0.0040$, stress $=0.045$ ). $\mathbf{C}$, Washington Navel orange (PERMANOVA, $R^{2}=0.26, P=0.0600$, stress $\left.=0.138\right)$. Grey $=$ lemon control $(n=11)$; black $=$ CLas $(+)$ lemon $(n=6)$; light blue $=$ navel control $(n=4)$; dark blue $=$ CLas(+) navel $(n=4)$. 
metabolites, or that, perhaps, the metabolome is not the primary driver of the community structure. To our knowledge, no other study has compared the root microbiome of different citrus varieties with an identical rootstock. This finding is supported by recent work involving apple fruit trees, which showed endophytic microbial community composition diverging as the distance in the pedigree grows (Liu et al. 2018). A limitation to our study was the relatively small sample size, particularly for navels, which likely offered insufficient power to detect statistically significant differences. This was underscored by the greater number of significant findings in the lemon group compared with navels. Nevertheless, significant effects were observed in both the metabolome and microbiome, and these results, coupled with the calculated effect sizes, were used to interpret the findings of this study. Another limitation was the lack of data regarding root exudate metabolites, which would have enabled stronger conclusions regarding plant crosstalk with microbial communities.

Response of Lisbon lemon to CLas. Few studies have examined the response of Citrus limon to CLas, and none has combined metabolomics with an analysis of the microbiome. Unique features of the lemon root metabolome included reductions in quinic acid, malic acid, GABA, methanol, ethanol, cytidine, synephrine, and several amino acids. GABA is a nonprotein amino acid that accumulates in plant tissue under stressed conditions, and it is synthesized in plants, animals, bacteria, and fungi via the GABA shunt pathway (Roberts 2007). Although its role in plant defense is unclear, it may act as a signaling molecule between plants and other organisms (Roberts 2007), and it has been shown to modulate plant growth by regulating the aluminum-activated malic acid transporter (ALMT) protein complex (Ramesh et al. 2015). Interestingly, reduced GABA concentrations in lemon occurred in concert with a reduction in malic acid. A similar effect was not observed for navels. Ethanol can increase in diseased plants because of the increasingly hypoxic conditions created by accumulating necrotic plant tissue (Kelsey and Joseph 1998); however, ethanol is also metabolized by plant tissues to synthesize organic acids, amino acids, lipids, and sugars to be utilized by the host (Cossins and Beevers 1963). Alterations in the microbial communities of lemon roots were primarily driven by a large significant reduction in Exophiala, which includes fungi that are endophytic and frequently colonize plant roots, as either a mutualist or a pathogen (Jumpponen 2001), possessing enzymes capable of breaking down polysaccharides (Caldwell et al. 2000).

Response of Washington Navel orange to CLas. Compared with lemon roots, fewer metabolites in navel roots were significantly altered in response to CLas, and fewer significant correlations between metabolite concentrations and CLas Ct values were observed. Interestingly, a greater number of bacterial and fungal genera appeared to be altered with CLas infection in navels compared with lemons. Despite the small sample size, the observed differences were sufficient to distinguish the root metabolome and microbiome of healthy and HLB-affected navel roots. Previously, it was shown that the metabolite signature of midribs from symptomatic 6-year-old CLas(+) Valencia orange leaves were distinguishable from asymptomatic CLas $(+)$ trees, but symptomatic
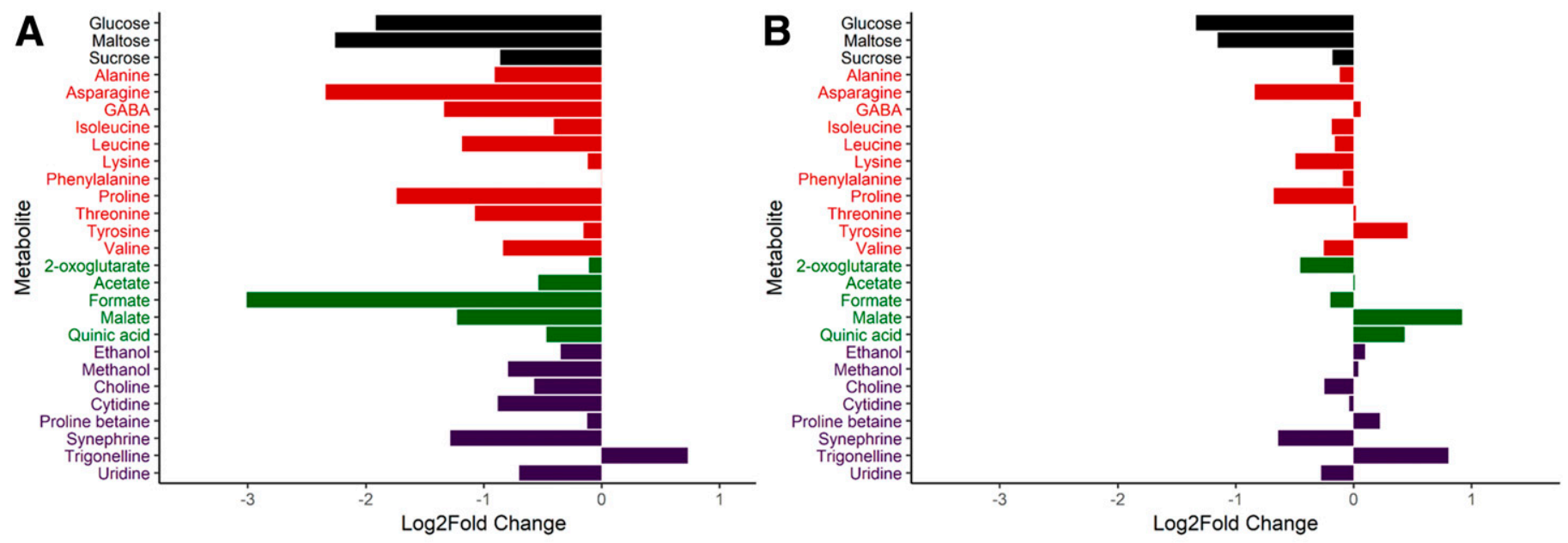

\section{C}

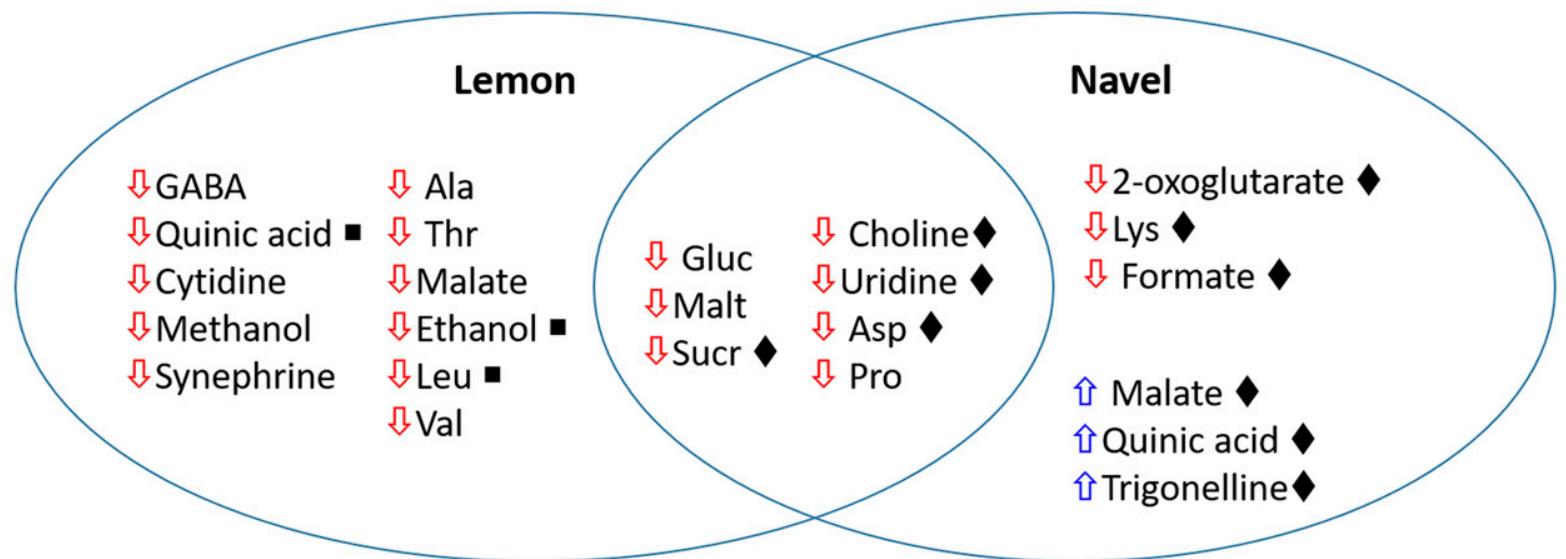

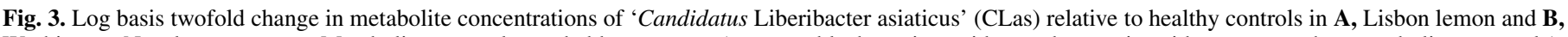

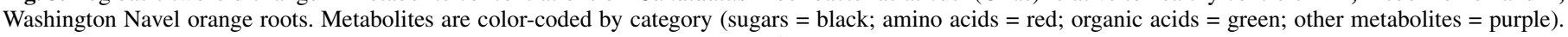

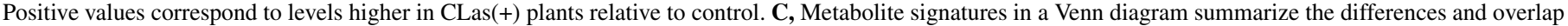
in the varietal response to CLas. Symbols indicate differences that were not significant but were associated with a large effect size. $\bullet=$ navel; $\mathbf{\bullet}=$ lemon. 
Family
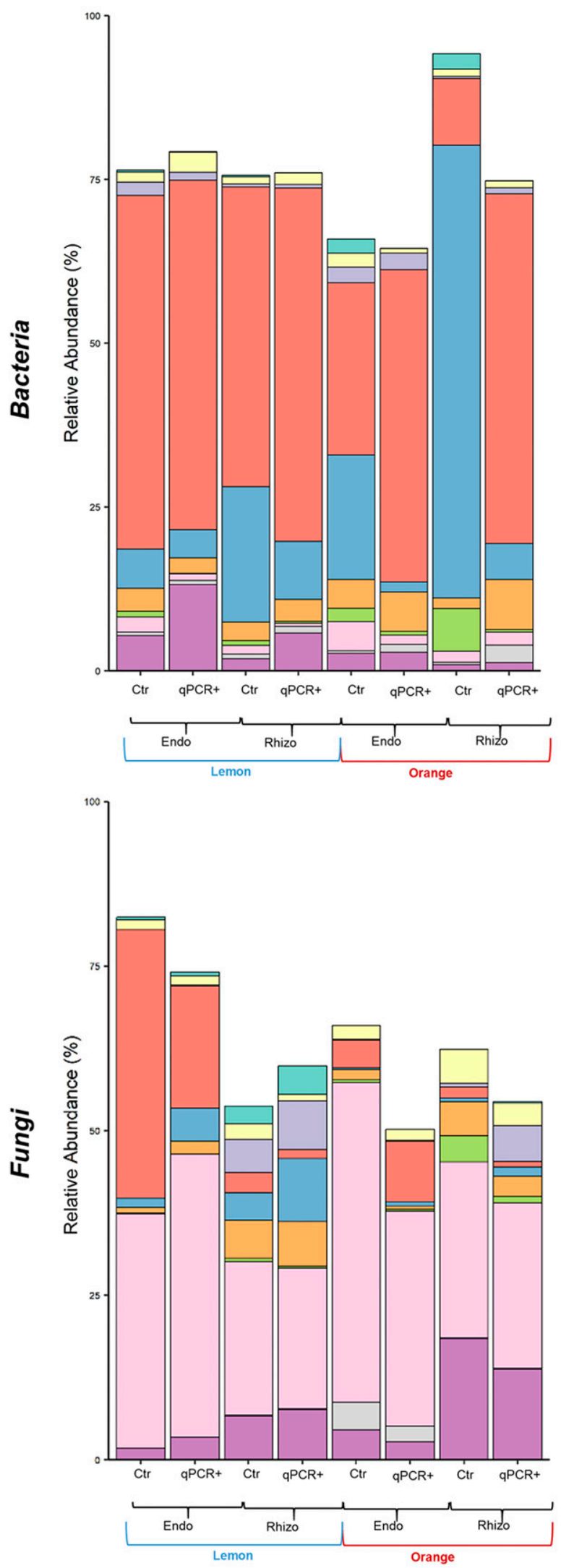

Genus
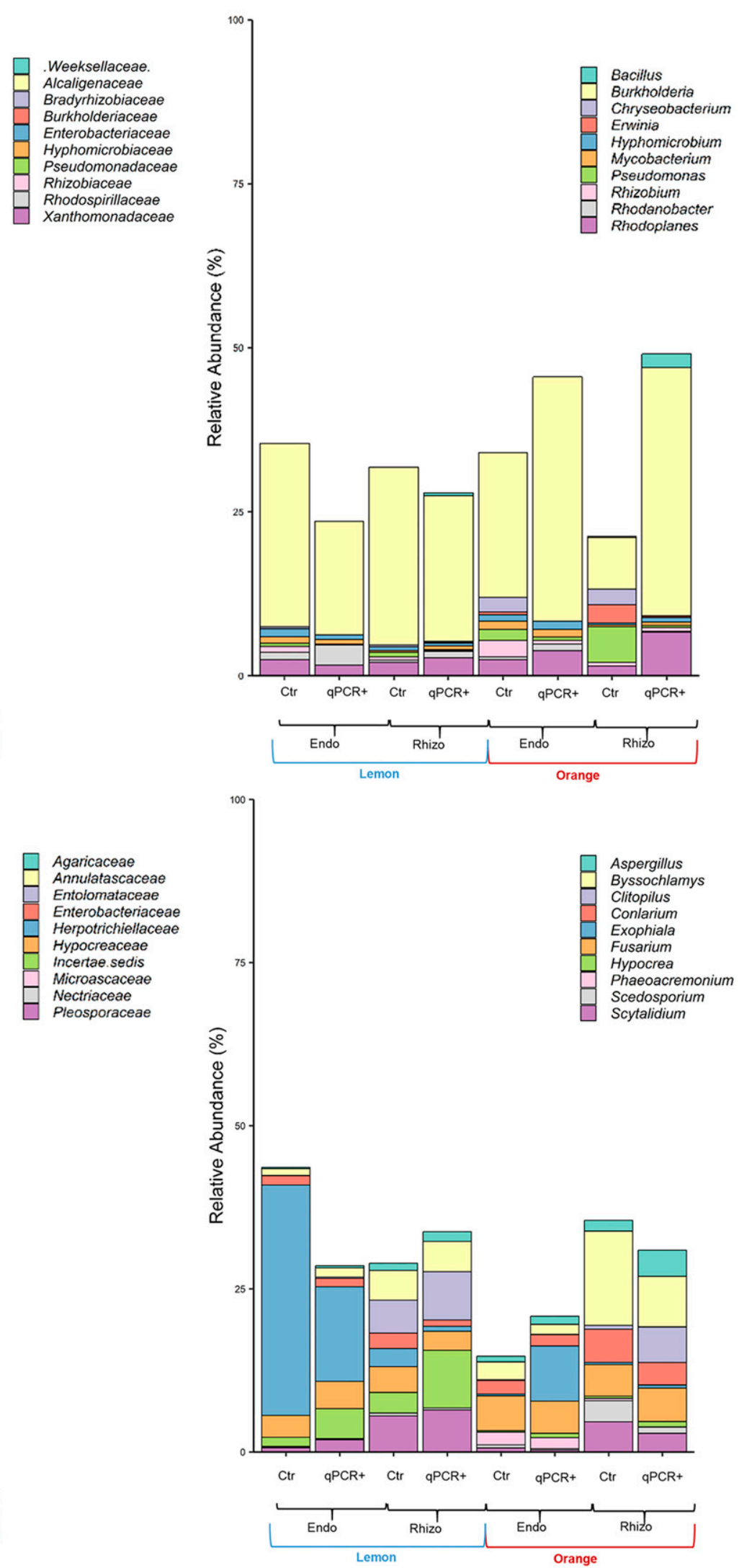

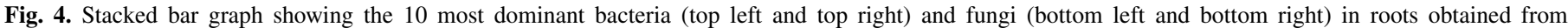
greenhouse-grown citrus trees. Values are median \% relative abundance. 'Candidatus Liberibacter asiaticus' trees are denoted as qPCR+. 
and asymptomatic root tissue were not (dos Santos Freitas et al. 2015). In the current study, Burkholderia was the dominant genus in both citrus varieties and increased in navel roots, but not lemon roots, in response to CLas. This contrasted with previous reports indicating that Burkholderia is associated with healthy sweet orange. For example, Burkholderia was observed to be more abundant in healthy 11-year-old mid-sweet orange trees compared with trees with HLB (Zhang et al. 2017), and in Valencia orange field trees affected by HLB (Trivedi et al. 2010). Burkholderiaceae has been identified as a key taxon in the root microbiome of healthy citrus (Zhang et al. 2017). However, Blaustein et al. (2017) reported higher abundances of Burkholderia in HLB-symptomatic compared with asymptomatic trees (Blaustein et al. 2017), which supported our findings of higher abundance in the roots of infected compared with healthy navels.

Trivedi et al. (2010) found that the relative abundance of Methylobacterium and Sphingobacterium genera increased in roots obtained from CLas(+) Valencia orange trees grown in a heavily infected citrus grove compared with uninfected trees. In contrast, Methylobacterium and Sphingobacterium were detected in the current study at low levels $(\leq 0.05 \%)$ in both healthy and CLas(+) lemon and navel roots and did not change with infection. Methylobacterium has been identified as a common source of microbial DNA contamination during sample preparation (Salter et al. 2014); therefore, it is possible that previous reports were spurious. Trivedi et al. (2010) also observed that several bacterial genera appeared to be associated with uninfected field-grown Valencia orange root samples (e.g., Caulobacter, Burkholderia, Lysobacter, Pantoea, Pseudomonas, Stenotrophomonas, Bacillus, Paenibacillus). Similar observations were not made in the current study of greenhouse-grown Washington Navel oranges, except for Pseudomonas, which was higher in healthy controls, and Bacillus, which increased in the rhizosphere of both varieties in response to CLas.

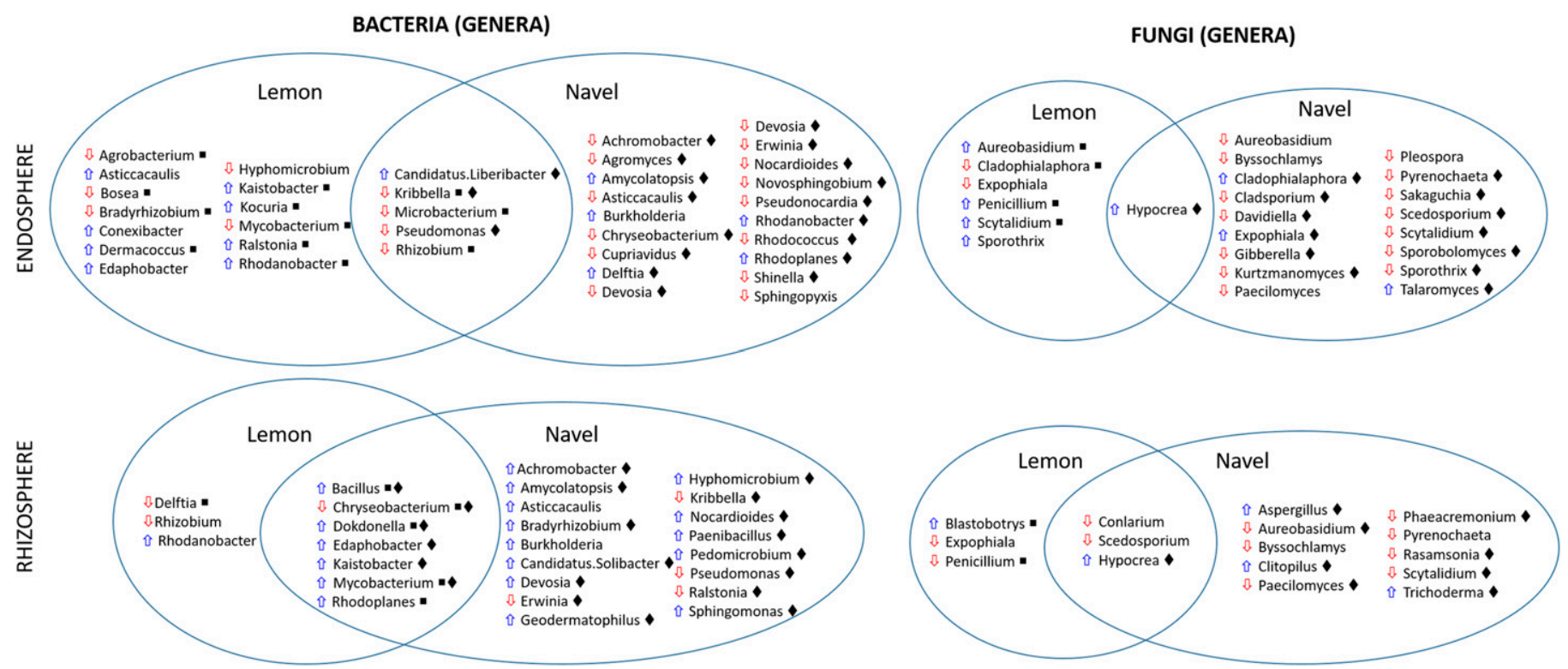

Fig. 5. Venn diagrams show bacterial and fungal signatures associated with 'Candidatus Liberibacter asiaticus' in the endosphere and rhizosphere of Lisbon lemon and Washington Navel orange roots. Symbols indicate differences that were not significant but were associated with a large effect size. $\bullet=$ navel; $\square=$ lemon.

A

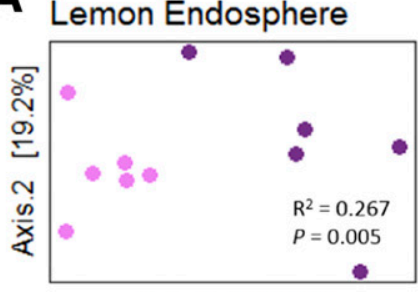

Axis.1 [30.8\%]

Lemon Rhizosphere

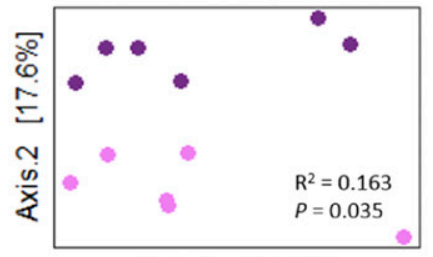

Axis.1 [29.7\%]

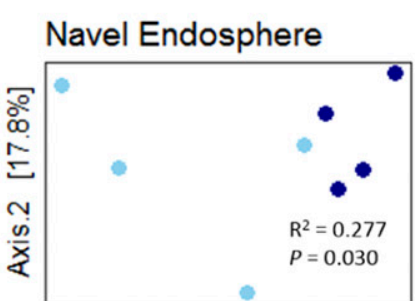

Axis.1 [38.7\%]

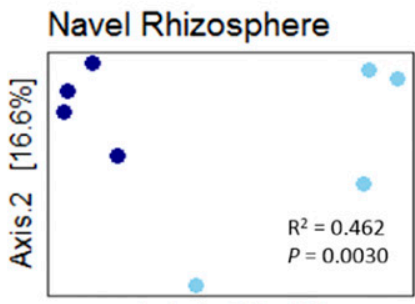

Axis.1 $[53.4 \%]$

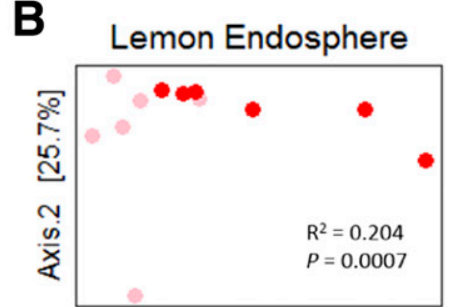

Axis.1 [35.1\%]

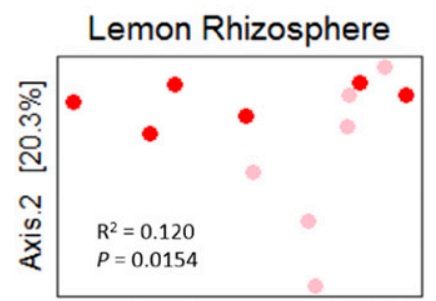

Axis.1 [22.4\%]

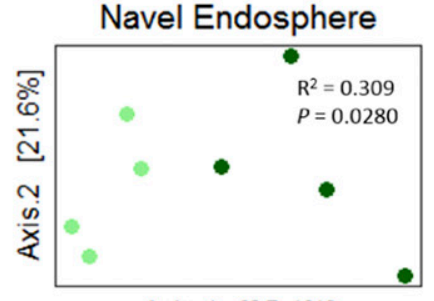

Axis.1 [35.4\%]

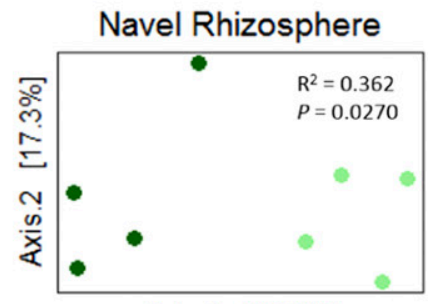

Axis.1 [39.6\%]

Fig. 6. Principal coordinate analysis (PCoA) plots showing differences in $\mathbf{A}$, bacterial and $\mathbf{B}$, fungal communities in Lisbon lemon and Washington Navel orange roots (PERMANOVA). Each point represents a single tree. Light circles are the control; dark circles are CLas $(+)$. Clustering was driven by infection status, particularly in navel samples despite a relatively smaller sample size. 
Similarities and differences in the responses of lemons and navels to CLas. There was overlap in the varietal response to CLas, including reductions in all sugars measured and in the amino acids proline and asparagine, and an increase in trigonelline. Furthermore, overlapping microbial shifts included decreases in Pseudomonas and Rhizobium, increases in Bacillus and Kaistobacter, and an increase in the fungi Hypocrea. Moreover, a dramatic decrease in Enterobacteriaceae was observed in both varieties. Recently, Enterobacteriaceae was shown to dominate the phyllosphere of arugula, thereby improving plant resistance to pathogen attacks through its antibiotic capabilities (Cernava et al. 2019). Altered sucrose concentrations in citrus roots were consistent with reports that CLas influences the carbohydrate gene expression in citrus host plants (Kim et al. 2009). Furthermore, starch accumulation in the leaf tissue and an extensive loss of starch reserves in the root system have been observed (Etxeberria et al. 2009).

Stress caused by pathogenic microorganisms alters the plant source/sink relationship (Lemoine et al. 2013); therefore, lower metabolite concentrations in the root system ('sink') are consistent with higher concentrations in citrus leaves ('source'). Proline is implicated in the plant defense response, and proline accumulation in source organs during a pathogen attack has been documented in several plant models (Qamar et al. 2015). Conversely, HLB appears to lower the concentrations of the amino acids alanine, proline, and threonine in fruit, the other dominant 'sink' organ of citrus (Slisz et al. 2012). Asparagine is involved in the transport and storage of nitrogen in plants. One study showed that asparagine concentrations in the roots of white lupin plants decrease rapidly when nitrogen availability in the surrounding environment becomes limited (Lea et al. 2007). Trigonelline is a secondary metabolite in niacin metabolism that accumulates and acts as an osmoprotectant during stress (Rajasekaran et al. 2001). Recently, the tgn gene cluster was identified in the degradation pathway of trigonelline to the end products succinate and methylamine, which are utilized by bacteria such as Agrobacterium, Burkholderia, and Pseudomonas as sources of carbon and nitrogen (Perchat et al. 2018).

$\mathrm{Xu}$ et al. (2018) showed that Burkholderia, Pseudomonas, and Rhizobium form part of the core citrus rhizosphere, and both Pseudomonas and Rhizobacterium are known to suppress plant pathogens and promote root growth (Preston 2004; Datta et al. 2015). In the current study, both lemons and navels responded to CLas infection with decreases in Pseudomonas and Rhizobium, whereas an increase in Burkholderia was observed only in navels. It is unclear why Burkholderia increased in navels in response to CLas, because others have associated this genus with beneficial effects in citrus (Trivedi et al. 2012; Zhang et al. 2017). However, Burkholderia is a large genus comprising many species, some of which that are beneficial and others that are pathogenic to plants (Eberl and Vandamme 2016).

Populations of Bacillus and Kaistobacter increased in both lemon and navel roots, whereas the fungus Hypocrea increased significantly in lemons. Members of Bacillus have been shown to colonize the roots and promote growth in tomato plants and Arabidopsis thaliana; interestingly, malic acid appears to act as a chemoattractant for these microbes (Lugtenberg and Kamilova 2009). Curiously, malic acid concentrations increased in navel roots and decreased in lemons, but large effect sizes corresponding to increased Bacillus in the rhizosphere of both citrus varieties were observed. Kaistobacter is associated with disease suppression in tobacco plants (Liu et al. 2016). Increases in these two potentially beneficial microbes suggested that Bacillus and Kaistobacter may form part of the global response to CLas in citrus. Hypocrea species have been shown to have biocontrol activity but also opportunistic pathogens that are often associated with rotting wood (Kubicek et al. 2008). Furthermore, it is possible that the abundance of Hypocrea increased in response to the advanced diseased state of HLB-affected plants, as observed in their decayed root mass relative to controls.
Other differences in the varietal response to CLas were evident. For instance, the concentrations of malic acid and quinic acid increased in navel roots, but they were decreased in lemons. Furthermore, several more metabolites in lemon were significantly different compared with navel. Quinic acid is an intermediate metabolite formed in the shikimate pathway, which plants and microbes utilize to synthesize the aromatic amino acids phenylalanine, tyrosine, and tryptophan, and it is important for protein biosynthesis and the production of plant hormones and secondary metabolites (Guo et al. 2014). No differences in the concentrations of phenylalanine and tyrosine in navel roots were observed (tryptophan was not detected), which suggested disruptions to the shikimate pathway did not occur, at least in terms of downstream products. However, bacteria, such as Bacillus, which increased in the rhizosphere of lemon and navel roots, are capable of metabolizing quinic acid into shikimic acid (Ghosh et al. 2014). Interestingly, large effect sizes for the metabolites 2-oxoglutarate, lysine, and formate were observed in navel but not lemon roots, despite the relatively smaller sample size in the former. This perhaps warrants further investigations to confirm these findings.

Dominant bacteria and fungi varied by root compartment (i.e., endosphere versus rhizosphere) and by variety. For example, Herpotrichiellaceae was the most abundant fungal family in the lemon endosphere, and it was reduced with CLas infection, but a similar observation was not made in the navel endosphere. A recent study identified a consortium of phloem-inhabiting bacteria in leaf tissue that appear integral to the survival of CLas, including Comamonadaceae, Flavobacteriaceae, Microbacteriaceae, and Pseudomonadaceae (Fujiwara et al. 2018). In the current study, Comamonadaceae was reduced in the endosphere and significantly increased in the rhizosphere of infected navel roots, which agreed with the results of Blaustein et al. (2017), who showed that a lower abundance of Comamonadaceae was associated with HLB-symptomatic compared with asymptomatic trees. Differences in Flavobacteriaceae were not observed, and the abundance of Microbacteriaceae was different in lemons compared with navels.

Tolerant versus susceptible varieties. A growing body of evidence points to a delineation among citrus varieties based on their tolerance or susceptibility to CLas. For example, varieties of sweet orange and grapefruit were shown to be highly sensitive to CLas infection, whereas Mexican limes and Eureka lemons appeared to be more tolerant (Folimonova et al. 2009), and some accessions of $C$. limon were shown to fare much better than sweet orange and mandarin in a published field study (Miles et al. 2017). Emerging evidence has suggested multiple genes involved in defense are upregulated to a greater extent in rough lemon compared with sweet orange, which may account for differences in the observed tolerance between the citrus species (Yu et al. 2017). Although some varieties of citrus within the citron pedigree appear to be more HLB-tolerant, Citrus sinensis and Citrus limon have been categorized as having approximately the same degree of susceptibility to CLas (Ramadugu et al. 2016); therefore, distinction based on tolerance and susceptibility may not be appropriate in the current study. However, Chin et al. (E. L. Chin, J. Ramsey, S. Saha, D. Mishchuk, J. Chaves, K. Howe, X. F. Zhong, M. FloresGonzalez, E. Mitrovic, M. L. Polek, K. Godfrey, L. A. Mueller, J. Bruce, M. Heck, and C. Slupsky, unpublished data) recently showed that the rate of CLas transmission was lower in C. limon, and that photosynthesis was less affected in presymptomatic plants compared with $C$. sinensis. In the root system, we observed varietal differences in the response to CLas characterized by larger changes in lemons in terms of the root metabolite composition, but greater differences in navels in terms of root microbial communities, which translated into distinct metabolite and microbial fingerprints emerging for each variety. The implication is that treatment targeting the health of the root system likely requires consideration for the varietal response to disease as opposed to a global approach to HLB management. 


\section{LITERATURE CITED}

Albrecht, U., Tripathi, I., Kim, H., and Bowman, K. D. 2019. Rootstock effects on metabolite composition in leaves and roots of young navel orange (Citrus sinensis L. Osbeck) and pummelo ( $C$. grandis L. Osbeck) trees. Trees (Berl.) 33:243-265.

Aleklett, K., Leff, J. W., Fierer, N., and Hart, M. 2015. Wild plant species growing closely connected in a subalpine meadow host distinct rootassociated bacterial communities. Peer J 3:e804.

Anderson, M. J. 2001. A new method for non-parametric multivariate analysis of variance. Austral Ecol. 26:32-46.

Blaustein, R.A., Lorca, G.L., Meyer, J.L., Gonzalez, C.F., Teplitski, M. 2017. Defining the core citrus leaf- and root-associated microbiota: Factors associated with community structure and implications for managing Huanglongbing (citrus greening) disease. Appl. Environ. Microbiol. 83:e00210-17.

Bodenhausen, N., Horton, M. W., and Bergelson, J. 2013. Bacterial communities associated with the leaves and the roots of Arabidopsis thaliana. PLoS One 8:e56329.

Bokulich, N. A., and Mills, D. A. 2013. Improved selection of internal transcribed spacer-specific primers enables quantitative, ultra-high-throughput profiling of fungal communities. Appl. Environ. Microbiol. 79:2519-2526.

Caldwell, B. A., Jumpponen, A., and Trappe, J. M. 2000. Utilization of major detrital substrates by dark-septate, root endophytes. Mycologia 92:230-232.

Caporaso, J. G., Kuczynski, J., Stombaugh, J., Bittinger, K., Bushman, F. D., Costello, E. K., Fierer, N., Gonzalez Peña, A., Goodrich, J. K., Gordon, J. L., Huttley, G. A., Kelley, S. T., Knights, D., Koenig, J. E., Ley, R. E., Lozupone, C. A., McDonald, D., Muegge, B. D., Pirrung, M., Reeder, J., Sevinsky, J. R., Turnbaugh, P. J., Walters, W. A., Widmann, J., Yatsunenko, T., Zaneveld, J., and Knight, R. 2010. QIIME allows analysis of highthroughput community sequencing data. Nat. Methods 7:335-336

Cernava, T., Erlacher, A., Sog, J., Sensen, C. W., Grube, M., and Berg, G. 2019. Enterobacteriaceae dominate the core microbiome and contribute to the resistome of arugula (Eruca sativa Mill.). Microbiome 7:13.

Cevallos-Cevallos, J. M., Rouseff, R., and Reyes-De-Corcuera, J. I. 2009. Untargeted metabolite analysis of healthy and Huanglongbing-infected orange leaves by CE-DAD. Electrophoresis 30:1240-1247.

Chelius, M. K., and Triplett, E. W. 2001. The diversity of archaea and bacteria in association with the roots of Zea mays L. Microbiol. Ecol. 41:252-263.

Chin, E. L., Godfrey, K., Polek, M., and Slupsky, C. 2017. ${ }^{1} \mathrm{H}$ NMR analysis of Citrus macrophylla subjected to Asian citrus psyllid (Diaphorina citri Kuwayama) feeding. Arthropod-Plant Interact. 11:901-909.

Chin, E. L., Mishchuk, D. O., Breksa, A. P., and Slupsky, C. M. 2014. Metabolite signature of 'Candidatus Liberibacter asiaticus' infection in two citrus varieties. J. Agric. Food Chem. 62:6585-6591.

Cossins, E. A., and Beevers, H. 1963. Ethanol metabolism in plant tissues. Plant Physiol. 38:375-380.

Cox, T. F., and Ferry, G. 1993. Discriminant analysis using non-metric multidimensional scaling. Pattern Recognit. 26:145-153.

Datta, A., Singh, R. K., Kumar, S., and Kumar, S. 2015. An effective and beneficial plant growth promoting soil bacterium "Rhizobium": A review. Ann. Plant Sci. 4:933-942.

dos Santos Freitas, D., Carlos, E. F., Soares de Souza Gil, M. C., Esteves Viera, L. G., and Alcantara, G. B. 2015. NMR-based metabolomic analysis of huanglongbing-asymptomatic and symptomatic citrus trees. J. Agric. Food Chem. 63:7582-7588.

Eberl, L., and Vandamme, P. 2016. Members of the genus Burkholderia: Good and bad guys. F1000 Res. 5:1007.

Etxeberria, E., Gonzalez, P., Achor, D., and Albrigo, G. 2009. Anatomical distribution of abnormally high levels of starch in HLB-affected Valencia orange trees. Physiol. Mol. Plant Pathol. 74:76-83.

Folimonova, S. Y., Robertson, C. J., Garnsey, S. M., Gowda, S., and Dawson, W. O. 2009. Examination of the responses of different genotypes of citrus to Huanglongbing (citrus greening) under different conditions. Phytopathology 99:1346-1354.

Fujiwara, K., Iwanami, T., and Fujikawa, T. 2018. Alterations of Candidatus Liberibacter asiaticus-associated microbiota decrease survival of $\mathrm{Ca}$. L. asiaticus in in vitro assays. Front. Microbiol. 9:3089.

Ghosh, A., Pawar, H., Pai, O., and Banerjee, U. C. 2014. Microbial transformation of quinic acid to shikimic acid by Bacillus megaterium. Bioresour. Bioprocess. 1:7.

Goedhart, J. 2016. Calculation of a distribution free estimate of effect size and confidence intervals using VBA/Excel. bioRxiv 073999. https://doi.org/ 10.1101/073999

Guo, J., Carrington, Y., Alber, A., and Ehlting, J. 2014. Molecular characterization of quinate and shikimate metabolism in Populus trichocarpa. J. Biol. Chem. 289:23846-23858.

Hamady, M., Walker, J. J., Harris, J. K., Gold, N. J., and Knight, R. 2008. Error-correcting barcoded primers for pyrosequencing hundreds of samples in multiplex. Nat. Methods 5:235-237.
Hu, L., Robert, C. A. M., Cadot, S., Zhang, X., Ye, M., Li, B., Manzo, D., Chervet, N., Steinger, T., van der Heijden, M. G. A., and Schlaeppi, K. 2018. Root exudate metabolites drive plant-soil feedbacks on growth and defense by shaping the rhizosphere microbiota. Nat. Commun. 9:2738.

Johnson, E. G., Wu, J., Bright, D. B., and Graham, J. H. 2014. Association of 'Candidatus Liberibacter asiaticus' root infection, but not phloem plugging with root loss on huanglongbing-affected trees prior to appearance of foliar symptoms. Plant Pathol. 63:290-298.

Jumpponen, A. 2001. Dark septate endophytes-are they mycorrhizal? Mycorrhiza 11:207-211.

Kelsey, R. G., and Joseph, G. 1998. Ethanol in Douglas-fir with black-stain root disease (Leptographium wageneri). Can. J. For. Res. 28:1207-1212.

Kim, J.-S., Sagaram, U. S., Burns, J. K., Li, J.-L., and Wang, N. 2009. Response of sweet orange (Citrus sinensis) to 'Candidatus Liberibacter asiaticus' infection: Microscopy and microarray analyses. Phytopathology 99: 50-57.

Kõljalg, U., Nilsson, R. H., Abarenkov, K., Tedersoo, L., Taylor, A. F. S., Bahram, M., Bates, S. T., Bruns, T. D., Bengtsson-Palme, J., Callaghan, T. M., Douglas, B., Drenkhan, T., Eberhardt, U., Dueñas, M., Grebenc, T., Griffith, G. W., Hartmann, M., Kirk, P. M., Kohout, P., Larsson, E., Lindahl, B. D., Lücking, R., Martín, M. P., Matheny, P. B., Nguyen, N. H., Niskanen, T., Oja, J., Peay, K. G., Peintner, U., Peterson, M., Põldmaa, K., Saag, L., Saar, I., Schüßler, A., Scott, J. A., Senés, C., Smith, M. E., Suija, A., Taylor, D. L., Telleria, M. T., Weiss, M., and Larsson, K.-H. 2013. Towards a unified paradigm for sequence-based identification of fungi. Mol. Ecol. 22:5271-5277.

Kubicek, C. P., Komon-Zelazowska, M., and Druzhinina, I. S. 2008. Fungal genus Hyporcrea/Trichoderma: from barcodes to biodiversity. J. Zhejiang Univ. Sci. B 9:753-763.

Lareen, A., Burton, F., and Schäfer, P. 2016. Plant root-microbe communication in shaping root microbiomes. Plant Mol. Biol. 90:575-587.

Lea, P. J., Sodek, L., Parry, M. A. J., Shewry, P. R., and Halford, N. G. 2007. Asparpagine in plants. Ann. Appl. Biol. 150:1-26.

Lemoine, R., La Camera, S., Atanassova, R., Dédaldéchamp, F., Allario, T., Pourtau, N., Bonnemain, J-L., Laloi, M., Coutos-Thévenot, P., Maurousset, L., Faucher, M., Girousse, C., Lemonnier, P., Parrilla, J., Durand, M. 2013. Source-to-sink transport of sugar and regulation by environmental factors. Front. Plant Sci. 4:272.

Li, W., Hartung, J. S., and Levy, L. 2006. Quantitative real-time PCR for detection and identification of Candidatus Liberibacter species associated with citrus Huanglongbing. J. Microbiol. Methods 66:104-115.

Liu, J., Abdelfattah, A., Norelli, J., Burchard, E., Schena, L., Droby, S., and Wisniewski, M. 2018. Apple endophytic microbiota of difference rootstock/ scion combinations suggests a genotype-specific influence. Microbiome 6: 18

Liu, X., Zhang, S., Jiang, Q., Bai, Y., Shen, G., Li, S., and Ding, W. 2016. Using community analysis to explore bacterial indicators for disease suppression of tobacco bacterial wilt. Sci. Rep. 6:36773.

Lugtenberg, B., and Kamilova, F. 2009. Plant-growth promoting rhizobacteria. Annu. Rev. Microbiol. 63:541-556.

McCollum, G., and Baldwin, E. 2016. Huanglongbing: devastating disease of citrus. Pages 315-361 in: Horticultural Reviews. 1st ed. J. Janick, ed., John Wiley \& Sons, Hoboken, NJ.

Melnyk, C. W. 2017. Plant grafting: Insights into tissue regeneration. Regeneration (Oxf.) 4:3-14.

Middlemis Maher, J., Markey, J. C., and Ebert-May, D. 2013. The other half of the story: Effect size analysis in quantitative research. CBE Life Sci. Educ. 12:345-351.

Miles, G. P., Stover, E., Ramadugu, C., Keremane, M. L., and Lee, R. F. 2017. Apparent tolerance to Huanglongbing in citrus and citrus-related germplasm. Hortic. Sci. (Prague) 52:31-39.

Nakagawa, S., and Cuthill, I. C. 2007. Effect size, confidence interval and statistical significance: A practical guide for biologists. Biol. Res. 82: 591-605.

Perchat, N., Saaidi, P.-L., Darii, E., Pellé, C., Petit, J.-L., Besnard-Gonnet, M., de Berardinis, V., Dupont, M., Gimbernat, A., Salanoubat, M., Fischer, C., and Perret, A. 2018. Elucidation of the trigonelline degradation pathway reveals previously undescribed enzymes and metabolites. PNAS 115:E4358E4367.

Preston, G. M. 2004. Plant perceptions of plant growth-promoting Pseudomonas. Philos. Trans. R Soc. Lond. B Biol. Sci. 359:907-918.

Qamar, A., Mysore, K. S., and Senthil-Kumar, M. 2015. Role of proline and pyrroline-5-carboxylate metabolism in plant defense against invading pathogens. Front. Plant Sci. 6:503.

R Core Team. 2012. R: A language and environment for statistical computing. $\mathrm{R}$ Foundation for Statistical Computing, Vienna, Austria. http://www. R-project.org/

Rajasekaran, L. R., Aspinalli, D., Jones, G. P., and Paleg, L. G. 2001. Stress metabolism. IX. Effect of salt stress on trigonelline accumulation in tomato. Can. J. Plant Sci. 81:487-498. 
Ramadugu, C., Keremane, M. L., Halbert, S. E., Duan, Y. P., Roose, M. L., Stover, E., and Lee, R. F. 2016. Long-term field evaluation reveals Huanglongbing resistance in citrus relatives. Plant Dis. 100:1858-1869.

Ramesh, S. A., Tyerman, S. D., Xu, B., Bose, J., Kaur, S., Conn, V., Domingos, P., Ullah, S., Wege, S., Shabala, S., Feijó, J. A., Ryan, P. R., and Gilliham, M. 2015. GABA signaling modulates plant growth by directly regulating the activity of plant-specific anion transporters. Nat. Commun. 6:7879.

Rastogi, G., Tech, J. J., Coaker, G. L., and Leveau, J. H. 2010. A PCR-based toolbox for the culture-independent quantification of total bacterial abundances in plant environments. J. Microbiol. Methods 83:127-132.

Roberts, M. R. 2007. Does GABA act as a signal in plants? Plant Signal. Behav. 2:408-409.

Romano, J., Kromrey, J. D., Coraggio, J., and Skowronek, J. 2006. Appropriate statistics for ordinal level data: Should we really be using t-test and Cohen's d for evaluating group differences on the NSSE and other surveys? Paper presented at the Annual Meeting of the Florida Association of Institutional Research.

Salter, S. J., Cox, M. J., Turek, E. M., Calus, S. T., Cookson, W. O., Moffatt, M. M., Turner, P., Parkhill, J., Loman, N., and Walker, A. W. 2014. Reagent and laboratory contamination can critically impact sequence-based microbiome analyses. BMC Biol. 12:87.

Slisz, A. M., Breksa, A. P., III, Mishchuk, D. O., McCollum, G., and Slupsky, C. M. 2012. Metabolomic analysis of citrus infection by 'Candidatus Liberibacter' reveals insight into pathogenicity. J. Proteome Res. 11: 4223-4230.
Trivedi, P., Duan, Y., and Wang, N. 2010. Huanglongbing, a systemic disease, restructures the bacterial community associated with citrus roots. Appl. Environ. Microbiol. 76:3427-3436.

Trivedi, P., He, Z., Van Nostrand, J. D., Albrigo, G., Zhou, J., and Wang, N. 2012. Huanglongbing alters the structure and functional diversity of microbial communities associated with citrus rhizosphere. ISME J. 6:363-383.

USDA-APHIS-PPQ-CPHST-S\&T. 2013. Multiplex TaqMan real-time PCR for screening detection of citrus Huanglongbing (HLB) bacteria in citrus tissues. USDA-APHIS-PPQ-CPHST-S\&T.

Wang, N., Stelinski, L. L., Pellz-Stelinski, K. S., Graham, J. H., and Zhang, Y. 2017. Tale of the huanglongbing disease pyramid in the context of the citrus microbiome. Phytopathology 107:380-387.

Xu, J., Zhang, Y., Zhang, P., Trivedi, P., Riera, N., Wang, Y., Liu, X., Fan, G., Tang, J., Coletta-Filho, H. D., Cubero, J., Deng, X., Ancona, V., Lu, Z., Zhong, B., Roper, M. C., Capote, N., Catara, V., Pietersen, G., Vernière, C., Al-Sadi, A. M., Li, L., Yang, F., Xu, X., Wang, J., Yang, H., Jin, T., and Wang, N. 2018. The structure and function of the global citrus rhizosphere microbiome. Nat. Commun. 9:4894.

Yu, Q., Chen, C., Du, D., Huang, M., Yao, J., Yu, F., Brlansky, R. H., and Gmitter, F. G., Jr. 2017. Reprogramming of a defense signaling pathway in rough lemon and sweet orange is a critical element of the early response to 'Candidatus Liberibacter asiaticus'. Hortic. Res. 4:17063.

Zhang, Y., Xu, J., Riera, N., Jin, T., Li, J., and Wang, N. 2017. Huanglongbing impairs the rhizosphere-to-rhizoplane enrichment process of the citrus rootassociated microbiome. Microbiome 5:97.

This article was modified on 19 Feb 2020. 
ERRATUM / Volume 109, Number 12, 2019 / PHYTO-03-19-0103-R

In the article "Metabolome and Microbiome Signatures in the Roots of Citrus Affected by Huanglongbing" by E. M. T. Padhi, N. Maharaj, S.-Y. Lin, D. O. Mishchuk, E. Chin, K. Godfrey, E. Foster, M. Polek, J. H. J. Leveau, and C. M. Slupsky, Figure 4 incorrectly indicates a drop in Hyphomicrobacteriaceae and an increase in Enterobacteriaceae instead of a dramatic drop in the bacterial family Enterobacteriaceae. The color legend for the fungal genera pertaining to Conlarium and Clitopilus has also been corrected due to a similar error in the color scheme. The corrected Figure 4 is shown below.

Revised Figure 4
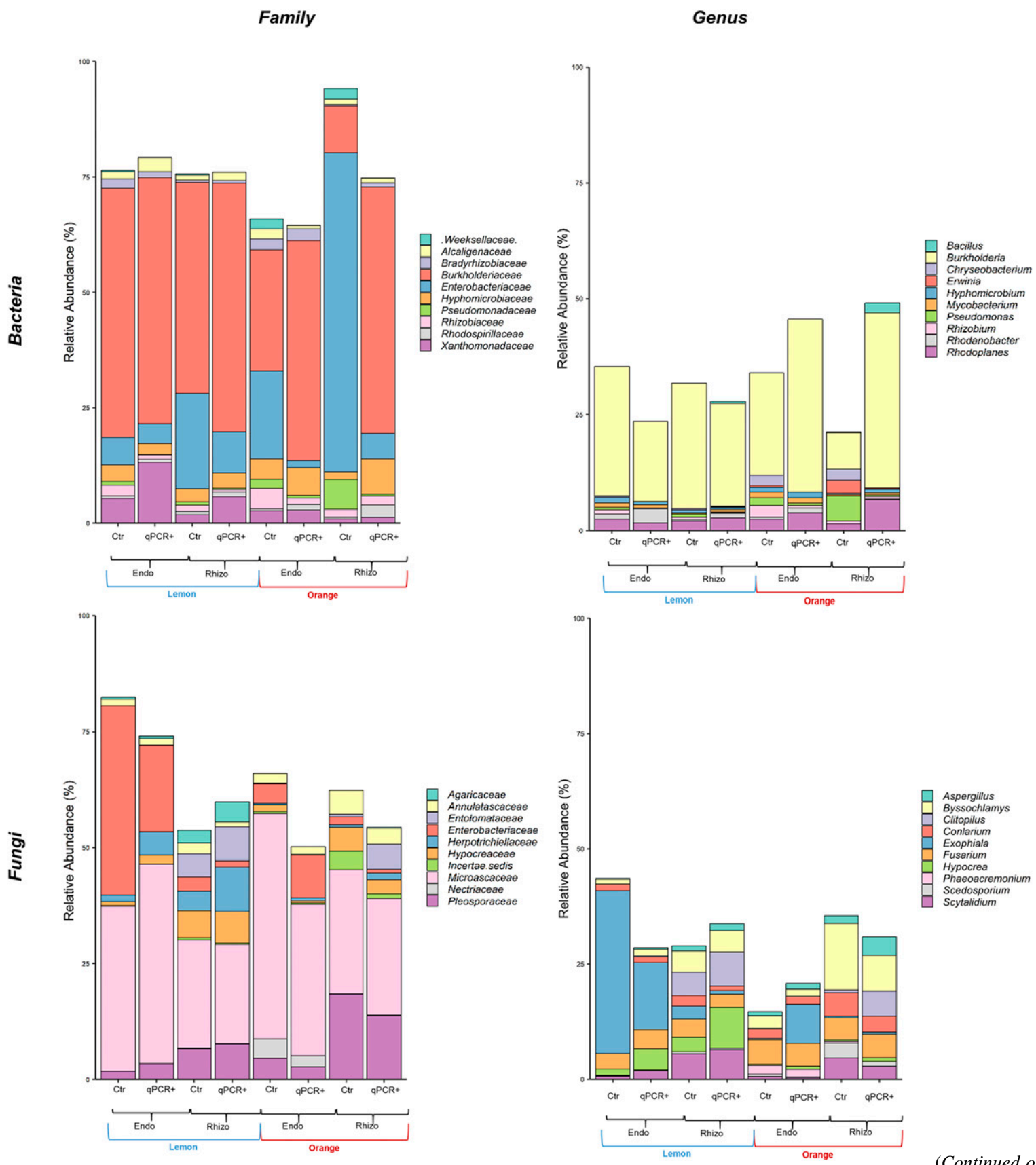

(Continued on next page)

Fig. 4. Stacked bar graph showing the 10 most dominant bacteria (top left and top right) and fungi (bottom left and bottom right) in roots obtained from greenhouse-grown citrus trees. Values are median \% relative abundance. 'Candidatus Liberibacter asiaticus' trees are denoted as qPCR+. 
Previously Published Version

Family
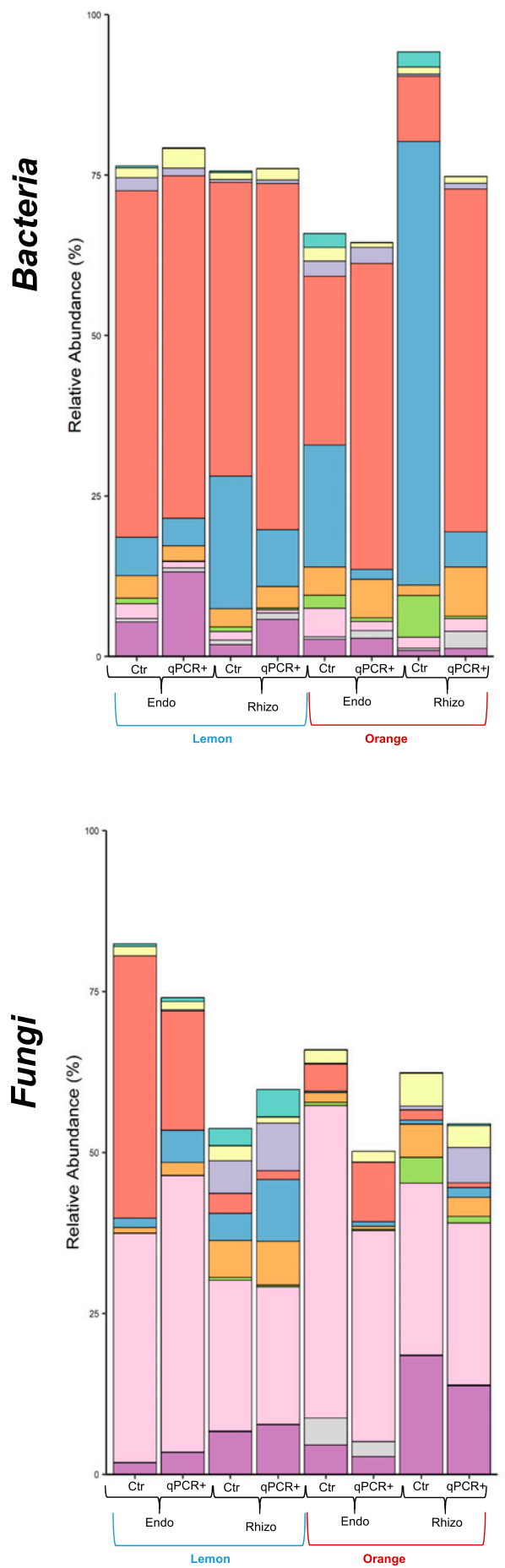

Genus
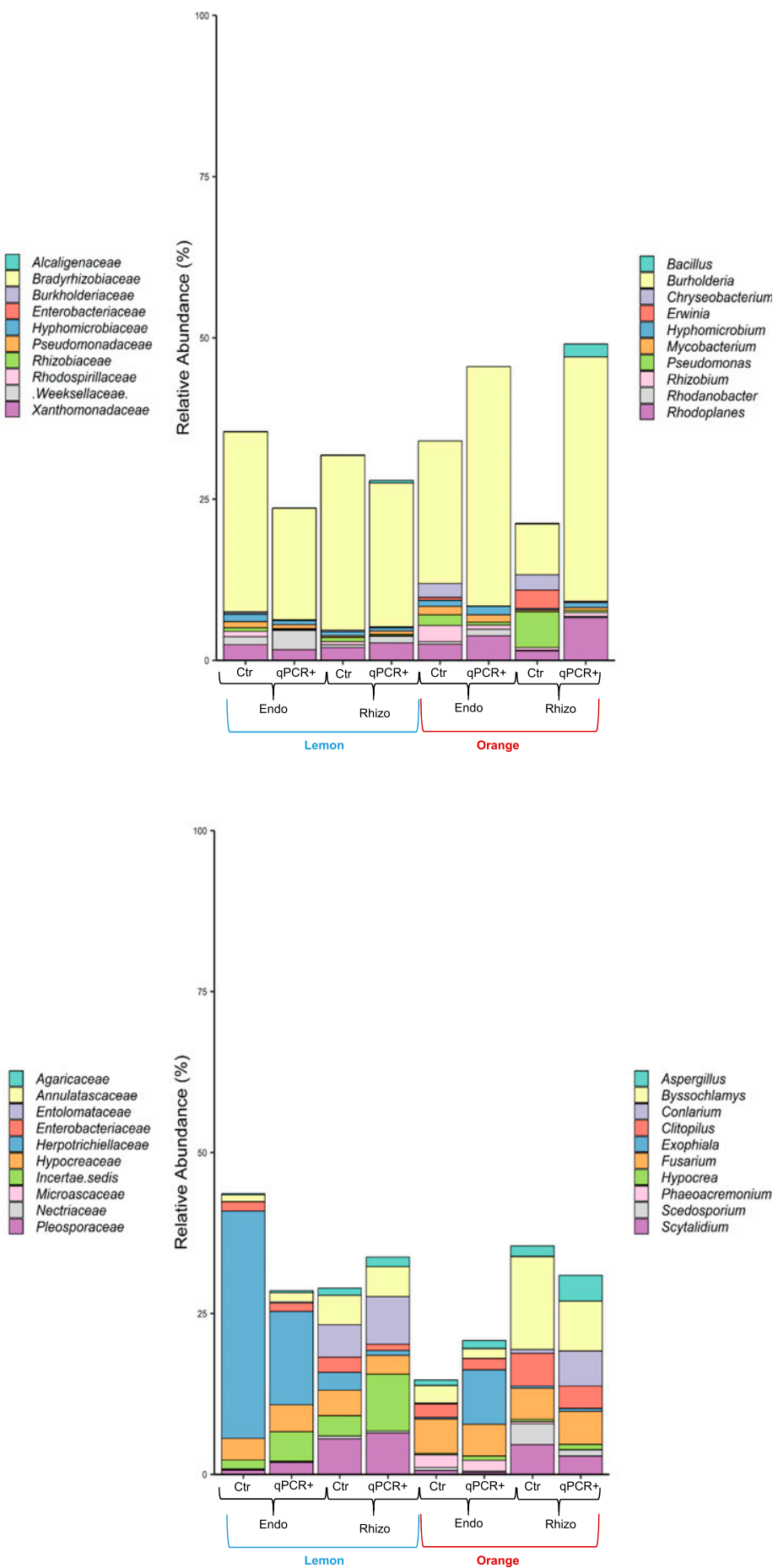

Fig. 4. Stacked bar graph showing the 10 most dominant bacteria (top left and top right) and fungi (bottom left and bottom right) in roots obtained from greenhouse-grown citrus trees. Values are median \% relative abundance. 'Candidatus Liberibacter asiaticus' trees are denoted as qPCR+. 